\title{
Deterministic Polynomial Time Algorithms for Matrix Completion Problems
}

\author{
Gábor Ivanyos * $\quad$ Marek Karpinski ${ }^{\dagger} \quad$ Nitin Saxena ${ }^{\ddagger}$
}

\begin{abstract}
We present new deterministic algorithms for several cases of the maximum rank matrix completion problem (for short matrix completion), i.e. the problem of assigning values to the variables in a given symbolic matrix as to maximize the resulting matrix rank. Matrix completion belongs to the fundamental problems in computational complexity with numerous important algorithmic applications, among others, in computing dynamic transitive closures or multicast network codings HKM05, HKY06. We design efficient deterministic algorithms for common generalizations of the results of Lovász and Geelen on this problem by allowing linear functions in the entries of the input matrix such that the submatrices corresponding to each variable have rank one. We present also a deterministic polynomial time algorithm for finding the minimal number of generators of a given module structure given by matrices. We establish further several hardness results related to matrix algebras and modules. As a result we connect the classical problem of polynomial identity testing with checking surjectivity (or injectivity) between two given modules. One of the elements of our algorithm is a construction of a greedy algorithm for finding a maximum rank element in the more general setting of the problem. The proof methods used in this paper could be also of independent interest.
\end{abstract}

\section{Introduction}

A linear matrix is a matrix having linear functions as its entries, say the linear functions are over a field $\mathbb{F}$ and in $\mathbb{F}\left[x_{1}, \ldots, x_{n}\right]$. The problem of maximum rank matrix completion, or just matrix completion for short, is the problem of assigning values from the field $\mathbb{F}$ to the variables $x_{1}, \ldots, x_{n}$ such that the rank of a given linear matrix is maximized (over all possible assignments). The notion of linear matrices appears in several places including both theory and applications, see HKM05, HKY06] for several references. The problem of matrix completion is a well studied problem, dating back to the work of Edmonds [Edm67] and Lovász [Lov79]. A similar problem (actually, more or less a special case) is nonsingular matrix completion, where we have a square linear matrix and we are interested in an assignment resulting in a nonsingular matrix. If the ground field is sufficiently large then the maximum

\footnotetext{
${ }^{*}$ Computer and Automation Research Institute of the Hungarian Academy of Sciences (MTA SZTAKI), Lágymányosi u. 11, 1111 Budapest. E-mail: Gabor.Ivanyos@sztaki.hu

${ }^{\dagger}$ Department of Computer Science and Hausdorff Center for Mathematics, University of Bonn, 53117 Bonn. E-mail: marek@cs.uni-bonn.de

${ }^{\ddagger}$ Hausdorff Center for Mathematics, Endenicher Allee 62, University of Bonn, 53115 Bonn. E-mail: ns@hcm.uni-bonn.de

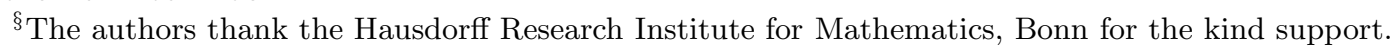


rank achieved by completion coincides with the rank of the linear matrix considered as a matrix over the function field $\mathbb{F}\left(x_{1}, \ldots, x_{n}\right)$, and hence, by standard linear algebra, finding a maximum rank completion (resp. determining the maximum rank) is in deterministic polynomial time reducible to instances of finding (resp. deciding the existence of) nonsingular completion of certain minors. Lovász gave an efficient randomized algorithm to find a matrix completion using the Schwartz-Zippel lemma [Sch80, Zip79], deducing that a random assignment of the variables will maximize the rank if the field is large enough (see also [IM83]). This is a method also useful in the fundamental problem of polynomial identity testing (PIT). Indeed matrix completion contains a special case of PIT: any arithmetic formula can be written as the determinant of a linear matrix [Val79], hence the formula would be zero iff the corresponding matrix could attain full rank (assuming a large enough field). Over large fields, this makes matrix completion an important problem in coRP as its derandomization would imply circuit lower bounds (see Kabanets \& Impagliazzo [KI03]).

Over small fields, matrix completion soon becomes a hard problem. This version has some important practical applications, for example in constructing multicast network codes [HKM05, and hence there are several results in the literature specifying the exact parameters for which the problem becomes NP-hard. The hardness of matrix completion and various related problems were first studied by Buss et al. [BFS99] and more recently by Harvey et al. HKY06]. In the former paper nonsingular matrix completion is proved to be NP-hard over fields of constant size, while the latter showed that matrix completion over the field $\mathbb{F}_{2}$ is NP-hard even if we restrict to a matrix where each variable occurs at most twice in its entries. This naturally raises the question: can we solve matrix completion by restricting the way the variables appear in the input matrix?

Few such cases are already known and they all look at mixed matrices, i.e. linear matrices where each entry is either a variable or a constant. Harvey et al. HKM05, building on the works of Geelen Gee99 and Murota Mur00, gave an efficient deterministic algorithm for matrix completion over any field if the mixed matrix has each variable appearing at most once. While Geelen at al. GIM03, GI05] gave an efficient deterministic algorithm when the mixed matrix is skew-symmetric and has each variable appearing at most twice.

In this paper we are interested in cases that are more general than the first case [HKM05. Consider a linear matrix $A \in \mathbb{F}\left[x_{1}, \ldots, x_{n}\right]^{m \times m}$ where the submatrix "induced" by each variable is of rank one, i.e. $A=B_{0}+x_{1} B_{1}+\cdots+x_{n} B_{n}$ where $B_{1}, \ldots, B_{n}$ are constant matrices of rank one (note that $B_{0}$ is also a constant matrix but of arbitrary rank). The case $B_{0}=0$ was first considered by Lovász in [Lov89], where it is shown how Edmonds' matroid intersection algorithm can be applied to solve this special case in deterministic polynomial time. The first main result in this paper is a common generalization of the results of Lovász [Lov89] and Geelen [Gee99]: we show that matrix completion problem for an arbitrary $B_{0}$ can be solved in deterministic polynomial time over any field:

Theorem 1.1. Let $\mathbb{F}$ be a field and let $B_{0}, \ldots, B_{n}$ be $m \times m$ matrices over $\mathbb{F}$. If $B_{1}, \ldots, B_{n}$ are of rank one then matrix completion for the matrix $\left(B_{0}+x_{1} B_{1}+\cdots+x_{n} B_{n}\right)$ can be done deterministically in poly $(m, n)$ field operations.

The proof of this theorem basically involves looking at the linear space $L:=\left\langle B_{0}, B_{1}, \ldots, B_{n}\right\rangle$ of matrices and showing that a greedy approach can be utilized to gradually increase the rank of an element in $L$. In fact our method is robust enough to check the rank maximality of a given matrix in $L$ without needing the rank one generators of $L$, they are needed only if we want to increase the rank (see Section 2). 
Matrix algebras or algebras of linear transformations (in this paper by an algebra we mean a linear space of matrices or linear transformations that is also closed under multiplication) play a crucial role in the algorithm for Theorem 1.1. We consider special instances of matrix completion problems where algebras of linear transformations arise naturally. These are certain module problems.

If $U$ and $V$ are vector spaces over the field $\mathbb{F}$ then we denote the vector space of linear maps from $U$ to $V$ by $\operatorname{Lin}(U, V)$. For $\operatorname{Lin}(U, U)$ we use the notation $\operatorname{Lin}(U)$. For simplicity, in this paper we consider modules over finite sets. (Actually, we work with modules over free associative algebras, however the main concepts and computational tasks we are concerned with can be understood without any knowledge from the theory of abstract associative algebras.) Let $\mathcal{S}$ be a finite set. A vector space $V$ over the field $\mathbb{F}$ equipped with a map $\nu$ from $\mathcal{S}$ into $\operatorname{Lin}(V)$ is called an $\mathbb{F}[\mathcal{S}]$-module (or an $\mathcal{S}$-module for short if $\mathbb{F}$ is clear from the context). We assume that the data for an $\mathcal{S}$-module is input by an $|\mathcal{S}|$-tuple of $\operatorname{dim} V$ by $\operatorname{dim} V$ matrices. In cases when the map $\nu$ is clear from the context - most typically when $\mathcal{S}$ is itself a set of linear transformations - we omit $\nu$ and denote the result $\nu(B) v$ of the action of $B \in \mathcal{S}$ on $v \in V$ by $B v$. For a set $\mathcal{S}^{\prime} \subseteq \operatorname{Lin}(V)$ of linear transformations the enveloping algebra $\operatorname{Env}\left(\mathcal{S}^{\prime}\right)$ is the smallest algebra containing $\mathcal{S}^{\prime}$. It is the linear span of finite products of transformations from $\mathcal{S}^{\prime}$

In the context of $\mathcal{S}$-modules the algebra $\mathcal{A}=\operatorname{Env}(\nu(\mathcal{S}) \cup I)$ is of special interest $(I$ is the identity in $\operatorname{Lin}(V))$. An $\mathcal{S}$-submodule of $V$ is a linear subspace closed under the action of all the transformations $\nu(B)$ corresponding to $B \in \mathcal{S}$. Obviously, the intersection of a family of submodules is again a submodule. In particular, if $T$ is a subset of $V$ then there is a smallest submodule of $V$ containing $T$ : the submodule generated by $T$. It is $\mathcal{A} T$, the linear span of vectors obtained by application of transformations from $\mathcal{A}$ to vectors from $T$. The set $T \subseteq V$ is a system of generators for the $\mathcal{S}$-module $V$ if $V=\mathcal{A} T$.

Cyclic submodules, i.e. those generated by a single element are of particular interest. For $v \in V$ we consider the map $\mu_{v}: \mathcal{A} \rightarrow V$ given by $\mu_{v}(B)=B v$. Obviously, $\mu_{v}$ is a linear map from $\mathcal{A}$ into $V$ and the set $\left\{\mu_{v} \mid v \in V\right\}$ is a linear space of linear maps from $\mathcal{A}$ to $V$. The rank of $\mu_{v}$ is the dimension of the submodule $\mathcal{A} v$ generated by $v$. The matrix completion problem in this context is finding an element $v$ which generates a submodule of maximum dimension. It turns out that this problem, which we call cyclic submodule optimization, is universal in matrix completion: there is a deterministic polynomial time reduction from maximum rank matrix completion to cyclic submodule optimization (over an arbitrary base field).

We show this universality in Section 3. Universality implies two hardness results. First, existence of a deterministic polynomial time algorithm for cyclic submodule optimization would imply deterministic solvability of the matrix completion problem over sufficiently large fields. Also, over small fields, cyclic submodule optimization is NP-hard. We also give analogous hardness results for existence of injective resp. surjective homomorphisms between modules (a $\mathcal{S}$-module homomorphism from $V$ to $V^{\prime}$ is a linear map in $\operatorname{Lin}\left(V, V^{\prime}\right)$ that commutes with the action of $\mathcal{S})$ :

Theorem 1.2. There is a deterministic polynomial time reduction from the existence of (resp. finding) a nonsingular matrix completion to the problem of checking for the existence of (resp. finding) a surjective (or injective) homomorphism between two modules.

This result is remarkable in view of the recent deterministic polynomial time algorithm of Brookbanks \& Luks [BL08] for module isomorphism problem (see also Chistov et al. [CIK97] over special base fields). 
In Section 4 we consider a problem which is in some sense "dual" to the cyclic submodule optimization. This is finding a system of generators of smallest size for a module. In contrast to hardness of the former problem, we have an efficient solution to the latter:

Theorem 1.3. Given a module structure on the $n$-dimension vector space $V$ over the field $\mathbb{F}$ in terms of $m n \times n$ matrices, one can find the minimum number of generators of $V$ deterministically using poly $(m, n)$ field operations.

Note that the above result includes testing cyclicity of modules efficiently over any field. This problem was considered in CIK97 over special fields. The algorithm is based on a greedy approach analogous to the method for Theorem 1.1 implicitly using certain submodule dimension optimization technique for a special class of (so called semisimple) modules.

\section{Matrix Completion with Rank One Matrices}

Let $V$ be a finite dimensional vector space over the field $\mathbb{F}$ and $L \leq \operatorname{Lin}(V)$ be a $\mathbb{F}$-linear space of linear transformations. Recall that $\operatorname{Env}(L)$, the enveloping algebra of $L$ is the linear span of products $h_{1} h_{2} \cdots h_{s}\left(s \geq 1, h_{1}, \ldots, h_{s} \in L\right)$. Obviously, $\operatorname{Env}(L)$ is also spanned by products of elements from an arbitrary basis of $L$. We will use the action of the enveloping algebra on the kernel of an idempotent transformation to optimize rank in a linear space, to that effect we present the following lemma. Its proof will also suggest how to greedily increment the rank.

Lemma 2.1. Let $V$ be a finite dimensional vector space over the field $\mathbb{F}$, let $L \leq \operatorname{Lin}(V)$ and assume that $e \in L$ is an idempotent $\left(e^{2}=e\right)$ such that $\mathrm{rk} e \geq \operatorname{rk} h$ for every $h \in L$. If $L$ is spanned by $e$ and certain rank one transformations, then $\operatorname{Env}(L)$ ker $e \subseteq e V$.

Proof. Assume, for contradiction, that $\operatorname{Env}(L)$ ker $e$ is not contained in $e V$. Then there exists a vector $v \in$ ker $e$ such that $L^{s} v \nsubseteq e V$ for some integer $s$. Let $s \geq 1$ be the smallest among such integers. Then there are matrices $h_{1}, \ldots, h_{s} \in L$ with $h_{s} \cdots h_{2} h_{1} v \notin e V$ such that for every $i$, the matrix $h_{i}$ is either $e$ or has rank one. Assume that $h_{j}=e$ for some $j \leq s$. Then $j>1$ as $e v=0$. Furthermore, the minimality of $s$ implies $h_{j-1} \cdots h_{1} v \in e V$, therefore, as $e w=w$ for every $w \in e V$, we have $h_{s} \cdots h_{j+1} h_{j} h_{j-1} \cdots h_{1} v=h_{s} \cdots h_{j+1} e h_{j-1} \cdots h_{1} v=$ $h_{s} \cdots h_{j+1} h_{j-1} \cdots h_{1} v$, contradicting the minimality of $s$. Thus all the matrices $h_{1}, \ldots, h_{s}$ are of rank one. Set $v_{0}=v$ and for $1 \leq i \leq s, v_{i}=h_{i} v_{i-1}$. The minimality of $s$ implies that for every $1 \leq i \leq s$ we have $v_{i} \in L^{i} v \backslash \sum_{j=0}^{i-1} L^{j} v$. In particular, the vectors $v_{0}, \ldots, v_{s}$ are linearly independent. Since $h_{i}$ is a rank one transformation on $V, h_{i} V=\mathbb{F} v_{i}(i=1, \ldots, s)$. From this, and from the minimality of $s$ we infer $h_{j} v_{i-1}=0$ for every $1 \leq i<j \leq s$ (otherwise $\left.v_{j} \in h_{j} L^{i-1} v \subseteq L^{i} v\right)$. We show below that $a:=e+h_{1}+\ldots+h_{s}$ is of a rank higher than $e$, leading to the desired contradiction.

Informally, we build a basis in which the matrix of $a$ is upper triangular. First we see that $a v_{i-1}=e v_{i-1}+\sum_{j<i} h_{j} v_{i-1}+v_{i}+\sum_{j>i} h_{j} v_{i-1}=v_{i}+e v_{i-1}+\sum_{j<i} h_{j} v_{i-1} \in v_{i}+e v_{i-1}+$ $\sum_{j<i} \mathbb{F} v_{j} \subseteq v_{i}+\left\langle v_{1}, \ldots, v_{i-1}\right\rangle(i=1, \ldots, s)$. (With some abuse of notation, for $i=1$, by $\left\langle v_{1}, \ldots, v_{i-1}\right\rangle$ we mean the zero subspace.) Hence the vectors $a v_{0}, \ldots, a v_{s-1}$ span the subspace $\left\langle v_{1}, \ldots, v_{s}\right\rangle$. Let $W$ be a direct complement to the subspace $\left\langle v_{1}, \ldots, v_{s-1}\right\rangle$ in $\mathrm{eV}$ and $w_{1}, \ldots, w_{t}$ be a basis of $W(t=\mathrm{rk} e-s+1)$. Then $a w_{i}=e w_{i}+\sum_{j=1}^{s} h_{j} w_{i}=w_{i}+\sum_{j=1}^{s} h_{j} w_{i} \in$ $w_{i}+\left\langle v_{1}, \ldots, v_{s}\right\rangle$. Therefore the vectors $a w_{1}, \ldots, a w_{t}$ are linearly independent even modulo the subspace $\left\langle v_{1}, \ldots, v_{s}\right\rangle$. Together with the fact that $\left\langle a v_{0}, \ldots, a v_{s-1}\right\rangle=\left\langle v_{1}, \ldots, v_{s}\right\rangle$, this implies that $\left\langle a v_{0}, \ldots, a v_{s-1}, a w_{1}, \ldots, a w_{t}\right\rangle=\left\langle v_{1}, \ldots, v_{s}, w_{1}, \ldots, w_{t}\right\rangle=\left\langle e V, v_{s}\right\rangle$. Thus the image of $a$ contains a subspace of dimension rk $e+1$ and hence rk $a \geq \operatorname{rk} e+1$, as claimed. 
In the proof above, the special case $s=1$ deserves special attention. In that case we have a simple method for increasing the rank over sufficiently large fields which works even without any assumption on the presence of rank one matrices. We will use this simple observation later in Section 4 .

Lemma 2.2. If $h, h^{\prime \prime} \in \operatorname{Lin}(V, U)$ are transformations such that $h^{\prime \prime} \operatorname{ker} h \nsubseteq h V$ then $h^{\prime}:=$ $h+\alpha h^{\prime \prime}$ will be of a higher rank than $h$ except for at most rk $h+1$ elements $\alpha \in \mathbb{F}$.

Proof. Let $k=$ rk $h$, and let $V_{0}$ be a subspace of $V$ complementary to ker $h$. Let $v_{1}, \ldots, v_{k}$ be a basis of $V_{0}$ and let $u_{1}, \ldots, u_{k}$ be a basis of the image $h V$. Choose a vector $v_{k+1}$ from ker $h$ such that $u_{k+1}:=h^{\prime \prime} v_{k+1} \notin h V$. Consider the matrix of the restriction of $h+x h^{\prime \prime}$ to $V_{0}+\mathbb{F} v_{k+1}$ in the bases $v_{1}, \ldots, v_{k}, v_{k+1}$ and $u_{1}, \ldots, u_{k}, u_{k+1}$. The last row and column of the constant term (the matrix of $h$ ) is zero while the lower right entry of the linear term (the matrix of $x h^{\prime \prime}$ ) is $x$. Expanding by the last row, we obtain that the linear term of the determinant of this $k+1$ by $k+1$ matrix is $d x$, where $d \neq 0$ is the determinant of the upper left $k \times k$ block of $h$. Thus the determinant is a nonzero polynomial in $x$ of degree at most $k+1$ and hence the corresponding $k+1$ by $k+1$ block of $h^{\prime}=h+\alpha h^{\prime \prime}$ is nonsingular showing that $h^{\prime}$ has rank higher than $k$ unless $\alpha$ is a root of this polynomial.

We state below a simple fact about the linear spaces of matrices that is useful in providing a certificate for the rank maximality of a given matrix.

Fact 2.3. Let $L \leq \operatorname{Lin}(V, U)$, where $U$ and $V$ are finite dimensional spaces over the field $\mathbb{F}$. Then for every $h \in L$ we have $\mathrm{rk} h \leq \operatorname{dim} V-\max \{\operatorname{dim} W-\operatorname{dim} L W \mid W \leq V\}$.

Proof. For any subspace $W \leq V$ pick a direct complement $W^{\prime}$ of $W$ in $V$. Now $\operatorname{dim} V-\operatorname{rk} h$ $=\operatorname{dim} V-\operatorname{dim} h V \geq(\operatorname{dim} W-\operatorname{dim} h W)+\left(\operatorname{dim} W^{\prime}-\operatorname{dim} h W^{\prime}\right) \geq \operatorname{dim} W-\operatorname{dim} L W$.

Using Edmonds' Matroid Intersection Theorem, Lovász (Section 3, [Lov89]) has shown that equality holds provided that $h$ is of maximum rank and if $L$ is spanned by rank one matrices. We give the following algorithmic generalization to the case when $L$ is spanned by rank one matrices and an arbitrary rank matrix.

Theorem 2.4. Let $U$ and $V$ be two finite dimensional vector spaces over the field $\mathbb{F}$, let $L \leq \operatorname{Lin}(V, U)$ be given by a basis and let an $h \in L$ be also given. Suppose that $L$ is spanned by $h$ and certain (unknown) transformations of rank one.

1) Then there exists a deterministic polynomial time algorithm which decides if $h$ is an element of $L$ of maximum rank. If $h$ is of maximum rank then a subspace $W$ of $V$ is constructed such that rk $h=\operatorname{dim} V-\max \{\operatorname{dim} W-\operatorname{dim} L W \mid W \leq V\}$.

2) If $h$ is not of maximum rank then, given rank one transformations that, together with $h$, span $L$, we can compute an element $h^{\prime} \in L$ with $\mathrm{rk} h^{\prime}>\mathrm{rk} h$ in deterministic polynomial time.

Proof. We may assume wlog that $\operatorname{dim} V=\operatorname{dim} U$, for otherwise we can pad transformations from $L$ with zeros to obtain a space $L^{\prime} \leq \operatorname{Lin}\left(V \oplus V^{\prime}, U \oplus U^{\prime}\right)$, where $\operatorname{dim} U \oplus U^{\prime}=\operatorname{dim} V \oplus V^{\prime}$ with some (possibly zero) spaces $U^{\prime}, V^{\prime}$. By padding a transformation $b \in \operatorname{Lin}(V, U)$ we mean the map $b^{\prime} \in \operatorname{Lin}\left(V \oplus V^{\prime}, U \oplus U^{\prime}\right)$ which is the direct sum of $b$ and the zero map: $b^{\prime}\left(v, v^{\prime}\right)=$ $(b v, 0)$.

Let $g: U \rightarrow V$ be an arbitrary nonsingular linear map such that $g h: V \rightarrow V$ is an idempotent. (The matrix of such a map $g$ can be obtained as the product of the matrices corresponding to the pivoting steps in Gaussian elimination for the matrix of $h$.) As $g$ is 
invertible, $h$ is of maximum rank within $L$ iff $g h$ is of maximum rank within $g L$. Also, rank one generators of $L$ are mapped to rank one generators of $g L$. If $g h$ is of maximum rank then by Lemma 2.1, $\operatorname{Env}(g L)$ ker $g h \leq g h V$. Conversely if $\operatorname{Env}(g L)$ ker $g h \leq g h V$ then, with $W_{0}:=\operatorname{Env}(g L) \operatorname{ker} g h$ and $W_{1}:=\operatorname{ker} g h$, we have $g L W_{0}, g L W_{1} \leq W_{0} \leq g h V$, and $W_{0} \cap W_{1}=0$ (if $v \in W_{0} \cap W_{1}$ then $v=g h u$ for some $u \in V$ and $g h v=0$, implying $0=$ ghghu= $g h u=v)$. Therefore with $W=W_{0}+W_{1} \leq V$ we have $g L W \leq W_{0}$ and $\operatorname{dim} V-\operatorname{rk} g h=$ $\operatorname{dim} W_{1}=\operatorname{dim} W-\operatorname{dim} W_{0} \leq \operatorname{dim} W-\operatorname{dim} g L W$. Now $g$ being invertible also implies that $\operatorname{dim} V-\operatorname{rk} h \leq \operatorname{dim} W-\operatorname{dim} L W$, which together with Fact 2.3 implies that $h$ has maximal rank. Thus if $\operatorname{Env}(g L)$ ker $g h \leq g h V$ then we can efficiently construct $W$ with the requested property, it is a witness of the maximality of the rank of $g h$ (resp. $h$ ) in $g L$ (resp. $L$ ). Thus, $h$ and hence $g h$ is not of maximum rank if and only if $\operatorname{Env}(g L)$ ker $g h$ is not contained in $g h V$. This can be decided in an obvious way.

Furthermore, if $L$ is spanned by $h$ and (known) rank one matrices $h_{1}, \ldots, h_{\ell}$ then the proof of Lemma 2.1 gives a linear combination of $g h$ and $g h_{1}, \ldots, g h_{\ell}$ of higher rank. Multiplying by $g^{-1}$ we obtain an element of $L$ of rank larger than rk $h$.

It is obvious that repeated applications of Theorem 2.4 completes the proof of Theorem 1.1

We remark that the shortest product $\Pi=g h_{1} \cdots g h_{\ell}$ with $\Pi$ ker $h \nsubseteq h V$ in the proof of Lemma 2.1 can be interpreted as a generalization of the notion of augmenting paths in the classical bipartite matching algorithms.

\section{Module Morphism Problems and Matrix Completion}

In this section we present hardness results of certain problems concerning modules. The key constructions are modules that we call bipartite modules as they resemble bipartite graphs.

\subsection{Bipartite modules}

Let $W_{1}$ and $W_{2}$ be two linear spaces over $\mathbb{F}$ and assume that we are given a linear subspace $R \leq \operatorname{Lin}\left(W_{1}, W_{2}\right)$ of linear maps from $W_{1}$ to $W_{2}$. We assume that $R$ is spanned by $\ell$ maps: $r_{1}, \ldots, r_{\ell}$. We consider the direct sum $W=W_{1} \oplus W_{2}$. We extend transformations $r \in R$ to linear transformations of $W$ by letting $r$ act on $W_{2}$ as the zero map. (That is, the extension maps $\left(w_{1}, w_{2}\right)$ to $\left(0, r w_{1}\right)$.) With some abuse of notation, we denote the extended map also by $r$ and consider $R$ as a subspace of $\operatorname{Lin}(W)$. Let $\mathcal{S}$ be the set $\left\{r_{1}, \ldots, r_{\ell}\right\}$ and the map $\nu: \mathcal{S} \rightarrow \operatorname{Lin}(W)$ defining the $\mathcal{S}$-module structure be just the identity map. This $\mathcal{S}$-module $W$ is a bipartite module.

\subsection{Universality of cyclic submodule optimization}

Assume that we are given a linear space $L$ of $\mathbb{F}$-linear maps from $U$ to $V$. It would be straightforward to consider the bipartite module $W$ for $W_{1}=U, W_{2}=V$ and $R=L$. However, this module does not turn out to be useful for our purposes and instead of it we consider another view: put $W_{1}=L, W_{2}=V, R=\left\{\mu_{u} \mid u \in U\right\}$, where $\mu_{u}(h)=h \cdot u$. Then, if $U$ is spanned by $u_{1}, \ldots, u_{\ell}$ then $\mathcal{S}$ is $\left\{\mu_{u_{1}}, \ldots, \mu_{u_{\ell}}\right\}$. Let $(h, v) \in W=L \oplus V$. Then the $\mathcal{S}$-submodule of $W$ generated by $(h, v)$ is $\mathbb{F}(h, v)+(0, h U)$ and its dimension is $1+$ rk $h$ if $h$ is not the zero map. Therefore this construction transforms matrix completion in $L$ to cyclic submodule optimization in $W$. 


\subsection{Module morphisms}

Let $U$ and $V$ be two $\mathbb{F}[\mathcal{S}]$-modules. An $\mathbb{F}$-linear map $\phi \in \operatorname{Lin}(U, V)$ is an $\mathcal{S}$-module homomorphism if for every $s \in \mathcal{S}$ and $u \in U$ we have $\phi(s u)=s \phi(u)$. The module homomorphism from $U$ to $V$ form a linear subspace $\operatorname{Hom}_{\mathbb{F}[\mathcal{S}]}(U, V)$ of $\operatorname{Lin}(U, V)$. Given the $\mathcal{S}$-module structure on $U$ and $V$ in terms of matrices over bases, a basis for the matrix space representing $\operatorname{Hom}_{\mathbb{F}[\mathcal{S}]}(U, V)$ can be computed with poly $(\operatorname{dim} U+\operatorname{dim} V+|\mathcal{S}|)$ field operations by solving a system of homogeneous linear equations.

It is not difficult to construct subspaces of $\operatorname{Lin}(U, V)$ which do not arise as spaces of module homomorphisms. Thus it is natural to ask how difficult are the matrix completion problems in spaces of module morphisms. It turns out that the cyclic submodules of a bipartite module $W$ (defined as $L \oplus V$ in the last subsection) arise as homomorphic images of another $\mathcal{S}$-module $W_{0}$, where $\mathcal{S}=\left\{r_{1}, \ldots, r_{\ell}\right\}$ and $W_{0}$ has basis $b_{0}, b_{1}, \ldots, b_{\ell}$ that by definition satisfy $r_{i} b_{0}=b_{i}$, $r_{i} b_{j}=0(i, j=1, \ldots, \ell)$.

This shows that hard matrix completion problems do arise in module morphism spaces. However, curiously enough, deciding existence and construction of module isomorphisms, i.e., module homomorphisms which are bijective linear maps can be accomplished in polynomial time (see [CIK97] with some restriction for the base field and [BL08] over arbitrary fields). We show that this is not the case for testing existence of injective or surjective module morphisms.

Module Injection: For the injective case, consider the bipartite modules $W$ and $W_{0}$ discussed above. The module $W_{0}$ is cyclic, it is generated by $b_{0}$. Therefore a module homomorphism is determined by the image of $b_{0}$. In this case for every pair $\left(w_{1}, w_{2}\right)$ there is indeed a homomorphism with $\psi\left(b_{0}\right)=\left(w_{1}, w_{2}\right)$. (For $i>0$ set $\psi\left(b_{i}\right)=\left(0, r_{i} w_{1}\right)$.) Consider the special case of the bipartite module $W$ used for showing hardness of cyclic submodule optimization: let $L$ be a space of linear maps from $U$ to $V$, put $W_{1}=L$ and $W_{2}=U$. Then the image of $W_{0}$ at the map $\psi$ under which the image of $b_{0}$ is $(h, v)$ is the subspace spanned by $(h, v),\left(0, h u_{1}\right), \ldots,\left(0, h u_{\ell}\right)$. This $\psi$ is injective if and only if $h$ is. This construction reduces both deciding and finding an injective transformation in $L$ (and also nonsingular matrix completion as special case) to deciding and finding an injective homomorphism from $W_{0}$ to $W$.

Module Surjection: Existence of and finding injective module morphisms can be transformed to the existence of resp. finding surjective morphisms between modules by standard dualization. If $M$ is a vector space over $\mathbb{F}$ then by $M^{*}$ we denote the space of (homogeneous) linear functions from $M$ to $\mathbb{F}$ (that is, $M^{*}=\operatorname{Lin}(M, \mathbb{F}$ ). If $\phi$ is an $\mathbb{F}$-linear map from the space $M_{1}$ to $M_{2}$ then the map $\phi^{*}: M_{2}^{*} \rightarrow M_{1}^{*}$ given as $\left(\phi^{*} f\right) v=\phi(f v)$ is again a linear map. (Note that if $\phi$ is interpreted as multiplication of column vectors by a matrix from the left then $\phi^{*}$ can be interpreted as multiplication of row vectors by the transposed matrix from the right.) Furthermore, if both $M_{1}$ and $M_{2}$ are finite dimensional then $\phi$ is injective (resp surjective) if and only if $\phi^{*}$ is surjective (resp. injective). If $M_{1}$ and $M_{2}$ are $\mathcal{S}$-modules given by the maps $\nu_{1}$ and $\nu_{2}$, then $\nu_{1}^{*}$ and $\nu_{2}^{*}$ given as $\nu_{i}^{*}(s)=\nu_{i}(s)^{*}$ make $M_{1}^{*}$ and $M_{2}^{*} \mathcal{S}$-modules. Furthermore, the linear map $\phi \in \operatorname{Lin}\left(M_{1}, M_{2}\right)$ is a module homomorphism from $M_{1}$ to $M_{2}$ if and only if $\phi^{*}$ is a module homomorphism from $M_{2}^{*}$ to $M_{1}^{*}$.

So when given vector spaces $U, V$ over $\mathbb{F}$, a linear subspace $L$ of $\operatorname{Lin}(U, V)$ with $\ell:=$ $\operatorname{dim} U$. We first construct modules $W$ and $W_{0}$ as in the previous reduction, so that the module homomorphism $\psi$ from $W_{0}$ to $W$ is injective if and only if $h \in L$ is an injective map where $\psi\left(b_{0}\right)=(h, v)$. Therefore $\Psi \in \operatorname{Hom}_{\mathbb{F}[\mathcal{S}]}\left(W^{*}, W_{0}^{*}\right)$ is surjective if and only if for the unique $\mathbb{F}$-linear map $\psi: W_{0} \rightarrow W$ such that $\Psi=\psi^{*}$ we have that $h$ is injective, where $\psi\left(b_{0}\right)=(h, v)$. This completes the proof of Theorem 1.2 . 


\section{Minimizing Number of Generators in Modules}

In this section we give an algorithm to minimize the number of generators in a given $\mathbb{F}[\mathcal{S}]$ module. It depends on a greedy property of the dimension of submodules in so called semisimple modules (which will be vaguely similar to that in Section 20). The radical of a module $V$ is the intersection of its maximal (more accurately, maximal proper) submodules. A finite dimensional module is semisimple if its radical is the zero submodule. Submodules, direct sums and factor modules of semisimple modules as well as the factor of a module by its radical are semisimple. (Recall that submodules, direct sums of spaces which are $\mathcal{S}$-modules inherit the $\mathcal{S}$-module structure in a natural way and so do factor spaces by submodules.)

In this section $V$ denotes a finite dimensional $\mathbb{F}[\mathcal{S}]$-module and $\mathcal{A}$ stands for the enveloping algebra $\operatorname{Env}(\nu(\mathcal{S}) \cup\{I\})$. For subsets $\mathcal{B} \subseteq \mathcal{A}$ and $U \subseteq V$ by $\mathcal{B} U$ we denote the linear span of the products $b u$, where $b \in \mathcal{B}$ and $u \in U$. In this context we omit braces around one-element sets. In particular, for $v \in V$, the submodule generated by $v$ is $\mathcal{A} v$.

\subsection{A Greedy Optimization of the Submodule Dimension in Semisimple Modules}

The annihilator $\operatorname{Ann}_{\mathcal{A}}(U)$ of $U \subseteq V$ is $\{a \in \mathcal{A} \mid a u=0$ for every $u \in U\}$. Note that the annihilator $\operatorname{Ann}_{\mathcal{A}}(v)$ of the single element $v \in V$ is just the kernel of the linear map $\mu_{v}: \mathcal{A} \rightarrow V$ given as $\mu_{v}(a)=a v$. The following lemma states that if the rank of $\mu_{v}$ is not maximal then we are in the situation of Lemma 2.2 .

Lemma 4.1. Assume that $V$ is semisimple. Then, for an arbitrary $u \in V$, $\operatorname{dim} \mathcal{A} u=$ $\max \left\{\operatorname{dim} \mathcal{A} u^{\prime} \mid u^{\prime} \in V\right\}$ iff $\operatorname{Ann}_{\mathcal{A}}(u) V \subseteq \mathcal{A} u$. Furthermore, if $\operatorname{Ann}_{\mathcal{A}}(u) V \nsubseteq \mathcal{A} u$ then an element $u^{\prime}$ with $\operatorname{dim} \mathcal{A} u^{\prime}>\operatorname{dim} \mathcal{A} u$ can be constructed using poly $(|\mathcal{S}|+\operatorname{dim} V)$ operations in $\mathbb{F}$.

The proof can be found in [CIK97]. For completeness, we also include a proof in the Appendix. The second part of the above lemma is especially interesting for small base fields where Lemma 2.2 does not apply. The next lemma can be used to give a generalization for submodules generated by larger systems.

Lemma 4.2. Assume that $V$ is semisimple. Then, for arbitrary positive integer $\ell$ and for elements $u_{1}, \ldots, u_{\ell} \in V, \operatorname{dim} \mathcal{A}\left\{u_{1}, \ldots, u_{\ell}\right\}=\max \{\mathcal{A} U \mid U \subseteq V, \# U \leq \ell\}$ if and only if for every $i \in[\ell]$, the $\mathcal{S}$-submodule generated by $u_{i}+W_{i}$ in the factor module $V / W_{i}$ is of maximum dimension, where $W_{i}$ denotes the submodule generated by $u_{1}, \ldots, u_{i-1}, u_{i+1}, \ldots, u_{\ell}$.

We relegate the proof of the lemma to the Appendix. The two lemmas above together with Lemma 2.2 immediately give the following.

Proposition 4.3. Let $v_{1}, \ldots, v_{n}$ be a basis of the semisimple $\mathbb{F}[\mathcal{S}]$-module $V$. Assume that $u_{1}, \ldots, u_{\ell}$ are elements of $V$ such that the submodule generated by $u_{1}, \ldots, u_{\ell}$ is not of maximum dimension among the submodules of $V$ generated by at most $\ell$ elements. If the $\mathbb{F}[\mathcal{S}]$-module structure on $V$ is given by an array of matrices, then we can find an index $i$ and construct $u_{i}^{\prime} \in V$ using poly $(|\mathcal{S}|+n)$ operations such that replacing $u_{i}$ with $u_{i}^{\prime}$ results in a submodule of larger dimension.

Furthermore, if $\mathbb{F}$ is of size larger than $n$ then there exist indices $i \in[\ell], j \in[n]$ such that replacing $u_{i}$ with $\left(u_{i}+\omega v_{j}\right)$ results in a submodule of larger dimension except for at most $n$ elements $\omega$ from $\mathbb{F}$. 
The above greedy property for the submodules of a semisimple module gives us the following technical lemma for general modules. It will be useful in the subsequent algorithm for optimizing the number of generators in any module without computing the radical explicitly.

Lemma 4.4. Let $v_{1}, \ldots, v_{n}$ be a basis of the $\mathcal{S}$-module $V$ which can be generated by $\ell$ elements and let $u_{1}, \ldots, u_{\ell}$ be elements of $V$ such that $U=\mathcal{A}\left\{u_{1}, \ldots, u_{\ell}\right\}<V$. If $W$ is a nonzero submodule such that $V=U \oplus W$ then there exist $i \in[\ell], j \in[n]$ such that for $U^{\prime}:=\mathcal{A}\left\{u_{1}, \ldots, u_{i}+\lambda v_{j}, \ldots, u_{\ell}\right\}, V=U^{\prime}+W$ but $U^{\prime} \cap W \neq\{0\}$ except for at most $2 n$ elements $\lambda \in \mathbb{F}$.

Proof. Let $U_{0}, W_{0}$ be the radicals of $U, W$ respectively. Let $V_{0}=U_{0} \oplus W_{0}$. Then the factor module $V / V_{0} \cong U / U_{0} \oplus W / W_{0}$ is semisimple and we can apply the preceding Proposition 4.3 to choose $i \in[\ell], j \in[n]$ such that the number of $\lambda$ 's, for which the dimension of $\left(U^{\prime}+V_{0}\right) / V_{0}$ is not larger than the dimension of $\left(U+V_{0}\right) / V_{0}$, is at most $\operatorname{dim} V / V_{0}$. Also for the same $i, j$ the $\lambda$ 's, for which $\left\{u_{1}, \ldots, u_{i}+\lambda v_{j}, \ldots, u_{\ell}\right\} \cup W$ do not span the whole of $V$, are the roots of a nonzero $\mathbb{F}$-polynomial of degree at most $\operatorname{dim} V$. Thus for this $i, j$ the number $\lambda$ 's, for which either $\operatorname{dim} U^{\prime} \leq \operatorname{dim} U$ or $V \neq U^{\prime}+W$, is at most $\operatorname{dim} V / V_{0}+\operatorname{dim} V \leq 2 n$.

\subsection{Algorithm for Finding $\ell$ Generators}

Using the previous Lemma, now we describe an iterative algorithm to find a minimal set of generators of a given module over a sufficiently large ground field.

Input: An $\mathcal{A}$-module $V$ given in terms of a set of generators. We assume that $\mathcal{A}$ is an $\mathbb{F}$ algebra where $|\mathbb{F}|>2 \operatorname{dim} V$.

Output: A set of at most $\ell$ elements generating $V$ over $\mathcal{A}$.

\section{Algorithm}

0) Initially pick any irredundant generating set $\left\{u_{1}, \ldots, u_{\ell}, u_{\ell+1}, \ldots\right\}$, set $U:=\mathcal{A}\left\{u_{1}, \ldots, u_{\ell}\right\}$ and $W:=\mathcal{A}\left\{u_{\ell+1}, \ldots\right\}$. Then $V=U+W$.

Outer loop:

1) Set $W^{\prime}:=U \cap W$.

2) If $W^{\prime}=W$, we are done and output $U$.

Inner loop:

3) apply Lemma 4.4 in $V / W^{\prime}$ to obtain $U^{\prime}$ generated by $\ell$ elements and satisfying:

$U^{\prime}+W=V$ and $\left(U^{\prime}+W^{\prime}\right) \cap W>W^{\prime}$.

4) If such a $U^{\prime}$ cannot be found then report " $\ell$ generators are insufficient for $V$ "; exit

Else set $U:=U^{\prime}$.

5) If $W \not \leq U+W^{\prime}$ then

continue inner loop with $W^{\prime}=\left(U+W^{\prime}\right) \cap W$.

6) Else

continue outer loop with $W=W^{\prime}$.

Analysis of the algorithm: At each step of the algorithm there is a pair $(U, W)$ of $\mathcal{S}$-modules such that $V=U+W$ and $U$ is known in terms of $\ell$ generators. At every repetition of the inner loop: $W^{\prime}$ becomes a larger submodule of $W$, since at Step 5 we know (from Step 3) that $\left(U+W^{\prime}\right) \cap W$ is strictly larger than $W^{\prime}$. At every repetition of the outer loop: $W$ becomes a smaller submodule of $V$, since at Step 6 we know (again from Step 3) that $W^{\prime}$ is strictly smaller than $W$. Thus, the number of times the algorithm can loop is bounded by $(\operatorname{dim} V)^{2}$, which easily makes the algorithm polynomial time. This gives a proof of Theorem 1.3 over large base fields. 
Over small base fields we use the algorithm of [FR85] or [CIW97] to compute the radical of $\mathcal{A}$ and the radical $V_{0}$ of $V$ therefrom and compute a minimal generating set $\Gamma_{0}$ of the factor module $V / V_{0}$ using Proposition 4.3 directly. For each $u_{0} \in \Gamma_{0}$ we pick a representative $u \in u_{0}+V_{0}$ and obtain a subset $\Gamma \subseteq V$ such that $|\Gamma|=\left|\Gamma_{0}\right|$ and $\Gamma \cup V_{0}$ generates $V$. By a standard property of the radical, we show that $\Gamma$ itself generates $V$. Indeed, let $U$ be the submodule generated by $\Gamma$. If $U \neq V$ then there is a maximal (proper) submodule $U^{\prime}$ containing $U$ and hence $\Gamma$. But $U^{\prime} \geq V_{0}$ by the definition of $V_{0}$, therefore $U^{\prime} \supseteq \Gamma \cup V_{0}$, a contradiction with that this union generates the whole $V$. This ends the proof of Theorem 1.3 .

\section{Concluding remarks}

We have shown that the maximum rank matrix in a linear space generated by rank one matrices and a further matrix of arbitrary rank can be found in deterministic polynomial time if the rank one generators are given. It would be interesting to know if there is an efficient deterministic method in the case where the rank one generators are not known. In this direction we have a deterministic polynomial time algorithm, which, given a matrix of maximum rank constructs a certificate that the rank is in fact maximal (see Theorem 2.4) without knowing the rank one generators. This implies that over sufficiently large base fields, the maximum rank matrix can be constructed in Las Vegas polynomial time. The best result of this flavor is the deterministic polynomial time algorithm of Gurvits Gur03, Gur04 which decides whether there exists a nonsingular matrix in the space generated by rational matrices under the assumption that the span over the complex numbers can be generated by unknown rank one matrices (with not necessarily rational entries). Unfortunately, this algorithm decides the mere existence of a nonsingular matrix without explicitly constructing one.

The space of the maps $\mu_{v}: \mathcal{A} \rightarrow V$ where $V$ is a semisimple $\mathcal{S}$-module and $\mathcal{A}$ is the corresponding enveloping algebra has a curious property that if $\mu_{v}$ is not of maximum rank there is a $v^{\prime \prime} \in V$ such that Lemma 2.2 applies for $h=\mu_{v}$ and $h^{\prime \prime}=\mu_{v^{\prime \prime}}$ (see Lemma 4.1). In particular, over a sufficiently large field $\mathbb{F}$ the rank of $\mu_{v}+\alpha \mu_{v^{\prime \prime}}$ will be higher for some $v^{\prime \prime}$ chosen from an arbitrary basis of $V$ and a "generic" $\alpha \in \mathbb{F}$.

It would be interesting to find more classes $\mathcal{L}$ of spaces of linear maps with such a "local rank incrementing" property: There is a constant $c$ such that for every $L \in \mathcal{L}$, if $h \in L$ is not of maximum rank then from an arbitrary basis $h_{1}, \ldots, h_{\ell}$ of $L$ one can choose maps $h_{i_{1}}, \ldots, h_{i_{c}}$, such that $h+\alpha_{1} h_{i_{1}}+\ldots+\alpha_{c} h_{i_{c}}$ has higher rank for some $\alpha_{1}, \ldots, \alpha_{c} \in \mathbb{F}$ (F is large enough.) 


\section{References}

[BFS99] J. F. Buss, G. S. Frandsen, and J. Shallit. The computational complexity of some problems of linear algebra. J. Comput. Syst. Sci., 58(3):572-596, 1999.

[BL08] P.A. Brooksbank and E.M. Luks. Testing isomorphism of modules. Journal of Algebra, 320(11):4020-4029, 2008.

[CIK97] A. Chistov, G. Ivanyos, and M. Karpinski. Polynomial time algorithms for modules over finite dimensional algebras. In ISSAC '97: Proceedings of the 1997 International Symposium on Symbolic and Algebraic Computation, pages 68-74, 1997.

[CIW97] A. M. Cohen, G. Ivanyos, and D. B. Wales. Finding the radical of an algebra of linear transformations. Journal of Pure and Applied Algebra, 117-118:177-193, 1997. (Proc. MEGA'96).

[Edm67] J. Edmonds. Systems of distinct representatives and linear algebra. Journal of Research of the National Bureau of Standards, 71B:241-245, 1967.

[FR85] K. Friedl and L. Rónyai. Polynomial time solutions of some problems of computational algebra. In STOC '85: Proceedings of the seventeenth annual ACM Symposium on Theory of Computing, pages 153-162, 1985.

[Gee99] J. F. Geelen. Maximum rank matrix completion. Linear Algebra and its Applications, 288:211-217, 1999.

[GI05] J. F. Geelen and S. Iwata. Matroid matching via mixed skew-symmetric matrices. Combinatorica, 25(2):187-215, 2005.

[GIM03] J. F. Geelen, S. Iwata, and K. Murota. The linear delta-matroid parity problem. Journal of Combinatorial Theory, Series B, 88(2):377-398, 2003.

[Gur03] L. Gurvits. Classical deterministic complexity of edmonds' problem and quantum entanglement. In STOC '03: Proceedings of the thirty-fifth annual ACM symposium on Theory of computing, pages 10-19, 2003.

[Gur04] L. Gurvits. Classical complexity and quantum entanglement. Journal of Computer and System Sciences, 69(3):448-484, 2004.

[HKM05] N. J. A. Harvey, D. R. Karger, and K. Murota. Deterministic network coding by matrix completion. In SODA '05: Proceedings of the sixteenth annual ACM-SIAM Symposium on Discrete Algorithms, pages 489-498, 2005.

[HKY06] N. J. A. Harvey, D. R. Karger, and S. Yekhanin. The complexity of matrix completion. In SODA '06: Proceedings of the seventeenth annual ACM-SIAM Symposium on Discrete Algorithms, pages 1103-1111, 2006.

[IM83] O. Ibarra and S. Moran. Probabilistic algorithms for deciding equivalence of straightline programs. JACM, 30(1):217-228, 1983.

[KI03] V. Kabanets and R. Impagliazzo. Derandomizing polynomial identity tests means proving circuit lower bounds. In STOC '03: Proceedings of the thirty-fifth annual ACM Symposium on Theory of Computing, pages 355-364, 2003. 
[Lov79] L. Lovász. On determinants, matchings and random algorithms. In FCT '79: Fundamentals of Computation Theory, pages 565-574, 1979.

[Lov89] L. Lovász. Singular spaces of matrices and their applications in combinatorics. Bol. Soc. Braz. Mat, 20:87-99, 1989.

[Mur00] K. Murota. Matrices and Matroids for Systems Analysis. Springer-Verlag, 2000.

[Pie82] R. S. Pierce. Associative Algebras. Springer-Verlag, New York, 1982.

[Sch80] J. T. Schwartz. Fast probabilistic algorithms for verification of polynomial identities. JACM, 27(4):701-717, 1980.

[Val79] L. G. Valiant. Completeness classes in algebra. In STOC 'r9: Proceedings of the eleventh annual ACM Symposium on Theory of Computing, pages 249-261, 1979.

[Zip79] R. Zippel. Probabilistic algorithms for sparse polynomials. Symbolic and Algebraic Computation, pages 216-226, 1979. 


\section{A Appendix}

\section{Algebras and modules}

In this part we summarize some basic notions and facts from the representation theory of algebras needed in the proof of Lemmas 4.1 and 4.2. For details, we refer the reader to the first few chapters of the textbook [Pie82].

Let $\mathbb{F}$ be an arbitrary field. An associative algebra with identity or algebra for short is a vector space $\mathcal{A}$ over $\mathbb{F}$ equipped with an associative $\mathbb{F}$-bilinear multiplication having a two-sided identity element $1_{\mathcal{A}}$ with respect to the multiplicative structure. If $V$ is a finite dimensional vector space of $\mathbb{F}$ then the $\mathbb{F}$ linear transformations of $V$ form a finite dimensional algebra $\operatorname{Lin}(V)$. Subalgebras of $\operatorname{Lin}(V)$, that is, subspaces closed under multiplication, containing the identity matrix are further examples. (In contrast to Section 2, where we considered algebras of linear transformations not necessarily having an identity, in this Appendix it will be convenient to consider algebras with identity only.) An algebra homomorphism from $\mathcal{A}$ to $\mathcal{B}$ is an $\mathbb{F}$-linear map $\phi: \mathcal{A} \rightarrow \mathcal{B}$ also satisfying $\phi\left(a_{1} \cdot a_{2}\right)=\phi\left(a_{1}\right) \cdot \phi\left(a_{2}\right)$ and $\phi\left(1_{\mathcal{A}}\right)=1_{\mathcal{B}}$.

A left $\mathcal{A}$-module or an $\mathcal{A}$-module for short is an $\mathbb{F}$-linear space $V$ equipped with a bilinear multiplication $: \mathcal{A} \times V \rightarrow V$ which commutes with the multiplication within $\mathcal{A}$ (that is, $\left.a_{1} \cdot\left(a_{2} \cdot v\right)=\left(a_{1} \cdot a_{2}\right) \cdot v\right)$ ). (In [Pie82, right modules are used. Here we we use left modules which are somewhat more common in the literature.) A module $V$ is unital if $1_{\mathcal{A}} v=v$ for every $v \in V$. All modules in this Appendix are assumed to be unital and finite dimensional over $\mathbb{F}$.

If $V$ is an $\mathcal{A}$-module then the map $\nu: \mathcal{A} \rightarrow \operatorname{Lin}(V)$ defined as $\nu(a) v=a \cdot v$ is a homomorphism from $\mathcal{A}$ into $\operatorname{Lin}(V)$. We say that $V$ is a faithful $\mathcal{A}$-module if the kernel of $\nu$ is zero, that is, if $a \in \mathcal{A}$ such that $a v=0$ for every $v \in V$ then $a=0$. If $\mathcal{S}$ is a finite set then $\mathbb{F}[\mathcal{S}]$, the algebra of noncommutative polynomials over $\mathbb{F}$ with indeterminates from $\mathcal{S}$ is an example of an infinite dimensional $\mathbb{F}$-algebra. It is the free algebra generated by $\mathcal{S}$ : if $\mathcal{A}$ is an algebra and $\nu$ is a map from $\mathcal{S}$ into $\mathcal{A}$ then $\nu$ can be extended to a unique algebra homomorphism from $\mathbb{F}[\mathcal{S}]$ to $\mathcal{A}$. In view of this, an $\mathbb{F}[\mathcal{S}]$-module structure on $V$ can be given by an arbitrary map $\nu: \mathcal{S} \rightarrow \operatorname{Lin}(V)$. Thus the notion of $\mathcal{S}$-module used in this paper is consistent with the notion of modules over free algebras.

A submodule of an $\mathcal{A}$-module is a linear subspace also closed under multiplication by elements of $\mathcal{A}$. The factor space of a submodule inherits the $\mathcal{A}$-module structure in a natural way and so do direct sums of linear spaces which are $\mathcal{A}$-modules. An $\mathcal{A}$ module $V$ is called simple if it has exactly two submodules: the whole $V$ and the zero submodule. The radical of a module is the intersection of its maximal (more precisely, maximal proper) submodules. A module $V$ is called semisimple if it is isomorphic to a direct sum of simple modules. By Section 2.7 of [Pie82, $V$ is semisimple if and only if its radical is the zero submodule. (Recall that in Section 4 we used this property as the definition for semisimplicity.) Furthermore, the factor module of $V$ by its radical is always semisimple. By Section 2.5 of [Pie82], the isomorphism classes of the constituents and their multiplicities in a decomposition of a semisimple module into a direct sum of simple modules are uniquely determined and, conversely, the isomorphism class of a direct sum of simple modules depend only on the isomorphism classes and multiplicities of the constituents. Direct sums and homomorphic images of semisimple modules are semisimple.

Let $V$ be a finite dimensional $\mathbb{F}[\mathcal{S}]$-module and let $\mathcal{A}$ be the enveloping algebra $\operatorname{Env}(I \cup \nu(\mathcal{S}))$ (the subalgebra of $\operatorname{Lin}(V)$ with the same identity generated by $\nu(\mathcal{S})$ ). Then $\mathcal{A}$ is the image of $\mathbb{F}[\mathcal{S}]$ under the unique algebra homomorphism from $\mathbb{F}[\mathcal{S}]$ to $\operatorname{Lin}(V)$ extending $\nu$ and $V$ is a 
faithful $\mathcal{A}$-module in the natural way. In this appendix we work with the $\mathcal{A}$-module structures of $V$, its submodules and factors as they coincide with the $\mathcal{S}$-module structures of the same objects. Assume that $V$ is semisimple. Then by Section 4.1 of [Pie82], $\mathcal{A}$ considered as a left module over itself by the algebra multiplication is also semisimple. Such algebras are called semisimple. Modules over semisimple algebras are semisimple, again by Section 4.1 of [Pie82]. (Recall that we consider only finite dimensional and unital modules.)

Let $\mathcal{A}$ be a semisimple algebra over $\mathbb{F}$ and let $\mathcal{A}$ as a left module over itself be isomorphic to the direct sum:

$$
\bigoplus_{i=1}^{t} V_{i}^{m_{i}},
$$

where $V_{i}$ are pairwise non-isomorphic $\mathcal{A}$-modules. Let $V$ be an $\mathcal{A}$-module. As $V$ is a homomorphic image of at most $\operatorname{dim} V$ copies of the module $\mathcal{A}$, we have

$$
V \cong \bigoplus_{i=1}^{t} V_{i}^{s_{i}},
$$

where the multiplicities $s_{i}$ are non-negative integers.

Lemma A.1. Let $\mathcal{A}$ and $V$ be as above and let $\ell$ be a positive integer. Let $U$ be a submodule of $V$ generated by $\ell$ elements. Then $U$ is of maximum dimension among the $\ell$-generated submodules of $V$ if and only if $U \cong \bigoplus_{i=1}^{t} V_{i}^{d_{i}}$ where $d_{i}:=\min \left(s_{i}, \ell m_{i}\right)$.

Proof. Let $W_{i}$ be the sum of all simple submodules of $V$ not isomorphic to $V_{i}$, Then the $\mathcal{A}$ module $V / W_{i}$ is isomorphic to $V_{i}^{s_{i}}$ and that the submodule dimension in $V$ is maximized iff it is maximized in $V / W_{i}$ for all $i \in[t]$. As a single generator in $V_{i}^{s_{i}}$ can generate a submodule of dimension at most that of $V_{i}^{\min \left(s_{i}, m_{i}\right)}$, we get that $\ell$ generators in $V_{i}^{s_{i}}$ can generate a submodule of dimension at most that of $V_{i}^{d_{i}}$. Repeating this for every $i \in\{1, \ldots, t\}$, we obtain that the maximum dimension is at most the dimension of the direct sum in the statement.

To see that this module occurs in fact as a cyclic submodule of $V$, let $W$ be the direct sum of $\ell$ copies of $\mathcal{A}$ (as a left $\mathcal{A}$-module) and let $w_{1}=\left(1_{\mathcal{A}}, 0, \ldots, 0\right), \ldots, w_{\ell}=\left(0, \ldots, 0,1_{\mathcal{A}}\right)$. Let $W_{0}$ be a submodule of $W$ isomorphic to $\bigoplus_{i=1}^{t} V_{i}^{\ell m_{i}-d_{i}}$ and let $V_{0}$ be a submodule of $V$ isomorphic to $\bigoplus_{i=1}^{t} V_{i}^{d_{i}}$. Then $V_{0} \cong W / W_{0}$ and $W / W_{0}$ is generated by $\ell$ elements: the images of $w_{1}, \ldots, w_{\ell}$ under the projection $W \rightarrow W / W_{0}$. Thus $V_{0}$ can be generated by the images of the latter $\ell$ elements under any isomorphism $W / W_{0} \cong V_{0}$.

\section{Proof of Lemma 4.1}

Let $V$ be a semisimple $\mathcal{S}$-module and let $\mathcal{A}=\operatorname{Env}(I \cup \nu(\mathcal{S}))$. Let $\mathcal{A}$ resp. $V$ be decomposed as in (11) resp. (2). Let $u \in V$. Assume that the dimension of the submodule $\mathcal{A} u$ is not maximal. Then, by Lemma A.1, there exists an index $i$ such that the multiplicity of $V_{i}$ in $\mathcal{A} u$ is less than both $s_{i}$ and $m_{i}$. Let $W$ be the submodule of $V$ which is the direct sum of the constituents of $V$ not isomorphic to $V_{i}$. Then $V / W \cong V_{i}^{s_{i}}$ and $V /(W+\mathcal{A} u)$ is isomorphic $V_{i}^{h}$ with some $h>0$. Recall that for a subset $X$ of $V$ the annihilator of $X$ in $\mathcal{A}$, denoted by $\operatorname{Ann}_{\mathcal{A}}(X)$ is $\{a \in \mathcal{A} \mid a x=0$ for every $x \in X\}$. Assume that $\operatorname{Ann}_{\mathcal{A}}(u) V \subseteq \mathcal{A} u$. Then every element of $\operatorname{Ann}_{\mathcal{A}}(u)$ act as zero on the factor module $V / \mathcal{A} u$ and hence also on the factor $V /(W+\mathcal{A} u)$. As the latter module is isomorphic to $V_{i}^{h}$ we obtain that $\operatorname{Ann}_{\mathcal{A}}(u) \subseteq \operatorname{Ann}_{\mathcal{A}}\left(V_{i}\right)$. Recall that the map $\mu_{u}: \mathcal{A} \rightarrow V$ is given as $\mu_{u}(a)=a u$. It is an $\mathcal{A}$-module homomorphism from the left 
module $\mathcal{A}$ to $V$. Its kernel is $\operatorname{Ann}_{\mathcal{A}}(u)$ and its image is $\mathcal{A} u$. Therefore $\mathcal{A} u \cong \mathcal{A} / \operatorname{Ann}_{\mathcal{A}}(u)$. Now $\operatorname{Ann}_{\mathcal{A}}\left(V_{i}\right)$ is also an $\mathcal{A}$-submodule of $\mathcal{A}$. Let $L$ be a submodule of $\mathcal{A}$ isomorphic to $V_{i}$. We claim that $L V_{i} \neq 0$. Indeed, if $L V_{i}=0$ then, by the assumed isomorphism, $L L=0$ as well, which is impossible by Section 3.2 of [Pie82]. The claim implies that the multiplicity of $V_{i}$ in $\operatorname{Ann}_{\mathcal{A}}\left(V_{i}\right)$ is zero and the same holds in $\operatorname{Ann}_{\mathcal{A}}(u) \subseteq \operatorname{Ann}_{\mathcal{A}}\left(V_{i}\right)$. But then the multiplicity of $V_{i}$ in the factor module $\mathcal{A} / \operatorname{Ann}_{\mathcal{A}}(u) \cong \mathcal{A} u$ is $m_{i}$. This contradiction finishes the proof of: if $\mathcal{A} u$ is not of maximum dimension then in fact $\operatorname{Ann}_{\mathcal{A}}(u) V \nsubseteq \mathcal{A} u$.

To see the reverse implication, assume that $\operatorname{Ann}_{\mathcal{A}}(u) V \nsubseteq \mathcal{A} u$ and let $w \in V$ and $b \in$ $\operatorname{Ann}_{\mathcal{A}}(u)$ such that $b w \notin \mathcal{A} u$. By Section 2.4 of [Pie82], there exists a submodule $W^{\prime}$ of $V$ such that $W^{\prime} \cap \mathcal{A} u=0$ and $W^{\prime}+\mathcal{A} u=V$. Write $w=a u+w^{\prime}$ where $a \in \mathcal{A}$ and $w^{\prime} \in W^{\prime}$. Put $u^{\prime}=u+w^{\prime}$. As $\mathcal{A} w^{\prime} \in W^{\prime}$, we have $\mathcal{A} u^{\prime}+W^{\prime}=\mathcal{A} u+W^{\prime}$. On the other hand, from $b w \notin \mathcal{A} u$ but $b a u \in \mathcal{A} u$ we infer that $b w^{\prime}$ is a nonzero element of $W^{\prime}$ and by the equality $b u^{\prime}=b u+b w^{\prime}=b w^{\prime}$, it is also an element of $\mathcal{A} u^{\prime}$. Therefore $\operatorname{dim} \mathcal{A} u^{\prime}>\operatorname{dim} V-\operatorname{dim} W^{\prime}=\operatorname{dim} \mathcal{A} u$, as required.

For a polynomial time implementation of the construction above, notice that a basis for $\operatorname{Ann}_{\mathcal{A}}(u)$ can be found by solving a system of linear equations. Then $b$ and $w$ can be found by testing membership of products of pairs of basis elements for $\operatorname{Ann}_{\mathcal{A}}(u)$ and those for $V$. To compute a direct complement of $\mathcal{A} u$, we first compute a projection $\pi$ of $V$ onto $\mathcal{A} u$ such that $\pi a=a \pi$ for every element of $\mathcal{A}$ (equivalently, for every element of a system of generators for $\mathcal{A}$, say $\nu(\mathcal{S})$ ). (Recall that a projection $\pi$ onto a subspace $V^{\prime}$ of $V$ is a map whose image is $V^{\prime}$ and it acts as the identity on $V^{\prime}$. If $W^{\prime}$ is submodule complementary to $\mathcal{A} u$ then the unique linear map which is the identity on $\mathcal{A} u$ and zero on $W^{\prime}$ is a projection onto $\mathcal{A} u$ which commutes with the action of $\mathcal{A}$ on $V$.) Once $\pi$ is constructed we take $\pi^{\prime}=I-\pi$. It is straightforward to see that the image $W^{\prime}=\pi^{\prime} V$ is in fact a direct complement of $\mathcal{A} u$. The element $w^{\prime}$ in the argument above is then just $\pi^{\prime} w$ and $u^{\prime}=u+\pi^{\prime} w$. This finishes the proof of Lemma 4.1,

\section{Proof of Lemma 4.2}

Let $V$ be a semisimple $\mathcal{S}$-module and let $\mathcal{A}=\operatorname{Env}(I \cup \nu(\mathcal{S}))$. Let $\mathcal{A}$ resp. $V$ be decomposed as in (11) resp. (2). Let $u_{1}, \ldots, u_{\ell} \in V$ and let $W_{i}=\mathcal{A}\left(\left\{u_{1}, \ldots, u_{\ell}\right\} \backslash\{i\}\right)$. As $\mathcal{A}\left\{u_{1}, \ldots, u_{\ell}\right\}=$ $\mathcal{A} u_{i}+W_{i}$, it is obvious that if, for some index $i$ there is an element $u_{i}^{\prime}$ such that modulo $W_{i}$, $\mathcal{A} u_{i}^{\prime}$ has a larger dimension than $\mathcal{A} u_{i}$, then replacing $u_{i}$ with $u_{i}^{\prime}$ results in a system generating a submodule of larger dimension.

To see the reverse implication let $W=\mathcal{A}\left\{u_{1}, \ldots, u_{\ell}\right\}$ and assume that for every $i$, the submodule of $V / W_{i}$ generated by $u_{i}+W_{i}$, that is, $W / W_{i}$ is a maximal dimensional cyclic submodule of $V / W_{i}$. Let $j \in\{1, \ldots, t\}$. By Lemma A.1. for every $i$, the multiplicity of $V_{j}$ in $W / W_{i}$ equals either the multiplicity of $V_{j}$ in $V / W_{i}$ or it is just $m_{j}$. If for some index $i$ the former is the case then the multiplicity of $V_{j}$ in $V / W$ is zero. Otherwise the multiplicity of $V_{j}$ in $W / W_{i}$ is $m_{j}$ for every index $i$. In the former case the multiplicity of $V_{j}$ in $W$ is the maximum possible among all submodules. Assume the latter case and let $U_{i j}$ denote the direct sum of the constituents of $\mathcal{A} u_{i}$ isomorphic to $V_{j}$. Then, for every index $i$, we have $U_{i j} \cap W_{i}=0$ and $U_{i j}$ is isomorphic to a direct sum of $m_{j}$ copies of $V_{j}$ as otherwise the multiplicity of $V_{j}$ in $W / W_{i}$ would be less than $m_{j}$. Thus $U_{i j}$ intersects $\sum_{i^{\prime} \neq i} U_{i^{\prime} j}$ trivially therefore they form an independent

system and hence $\sum_{i=1}^{\ell} U_{i j} \cong V_{j}^{\ell m_{j}}$ showing that the multiplicity of $V_{j}$ is optimal in this case as well. Repeating this for every irreducible module $V_{j}$, we obtain that the dimension of $W$ is indeed the maximum possible. This finishes the proof of Lemma 4.2 , 